\title{
A decoupled path-following control algorithm based upon the decomposed trajectory error
}

\author{
Hsin-Chiang Ho, Jia-Yush Yen*, Shui-Shong Lu \\ Department of Mechanical Engineering, National Taiwan University, Taipei, Taiwan 10764, Taiwan, ROC
}

Received 15 August 1998

\begin{abstract}
This paper proposes a new path-following control algorithm for the machine tool servo systems. The control system first decomposes the contouring error into the normal tracking error and the advancing tangential error. A dynamic decoupling procedure is then applied to the system dynamics. Finally, a dynamic decoupled controller is proposed to compensate the decomposed tangential and the normal tracking errors. The normal control minimizes the perpendicular tracking error while the tangential control maintains a desired feed rate. The proposed method is applied to the control of an experimental $x-y$ table. Experimental results show that the proposed control can achieve good tracking and trajectory following characteristics. The new algorithm also enables the design of a non-overshooting controller along the path. This will result in a no-overcutting process for the machine tool operation. (C) 1999 Published by Elsevier Science Ltd. All rights reserved.
\end{abstract}

Keywords: Machine tool control; Motion control; Servosystems

\section{Introduction}

Machine tool control systems maintain two control objectives: to follow a pre-specified trajectory as closely as possible, and to maintain a pre-specified advancing speed. These systems break the desired trajectory into small segments, and step along these segments to form the path. When the trajectories are multi-dimensional, the systems divide the motion into simultaneous movements in different axes [1]. The drivers of different axes have to move in synchronous with one another to obtain the desired trajectory. If the controllers operate independently from each

* Corresponding author. Tel. and fax: + 886-2-2366-0734; e-mail:jyen@ccms.ntu.edu.tw 
other, then any load disturbance or any difference in the performance of each axis may cause contour error [1].

Many researchers have addressed the issue of axis impedance matching [2-7]. Sarachik and Ragazzin [2] proposed the 'master-slave' structure in 1957 to enhance the contour following capability. In 1980, Koren [3] introduced the concept of cross-coupled control to reduce contour error. Tomizuka et al. [5-7] proposed different methods to synchronize the two axis motion, and more recently, Kulkarni and Svinivasan [8,9] formulated the linear-quadratic optimally crosscoupled control problem. There are also efforts based on variable-gain and adaptive control algorithms [10,11]. The basic concept behind these machine tool controls is to reduce the distance between the cutter location and the desired surface. However, the formulation for eliminating only this error would result in a standing regulation problem. Thus, all of these control algorithms are point-to-point algorithms to achieve motion along the desired path [12-14]. Traditionally, each axis has its own controller, which does not obtain information from other axes [3]. The newer controllers try to overcome this problem by introducing an additional feedback law derived from the path-following error in order to enhance the contour error rejection capability of the system.

In this paper, the contour error compensation is treated as a trajectory regulation problem. A good motion controller should achieve trajectory regulation at any time. On the other hand, the movement along the trajectory represents a speed regulation problem. It is thus natural to decouple the control action into a normal control action, which regulates the system around the desired trajectory, and a tangential control action, which manipulates the speed control problem. To achieve this purpose, one has to first decompose the tracking error into a normal and a tangential component. Separate control laws must then be applied to a dynamically decoupled system for independent tracking and feed rate control. Based on this argument, this paper thus proposes a new control algorithm, in which a dynamic decoupled controller operates on the decomposed error to achieve independent tracking and feed rate control. This design algorithm allows the design of a non-overshooting controller around the curve. In practice, it provides a way to design a machine tool control system with no overcutting operation.

\section{Reference commands generation}

The desired cutting trajectory can usually be represented in parametric form as $P_{\mathrm{d}}(x(u), y(u), z(u)), u \in[0, L][13]$. Assume that the position of the tool at some time is at $P_{\mathrm{i}}$ (Fig. $1)$. Because the target is the curve $P_{\mathrm{d}}$, errors in two separate directions are considered: a normal error and a tangential error. Ideally, the normal controller reduces the contour error; and the tangential controller maintains the desired feed rate, or cutting speed. Together, they achieve contouring accuracy.

The procedures to define the errors are divided into two parts: (1) define the projection point $P_{\mathrm{di}}$ and find the contour error $e_{\mathrm{c}}$, and (2) define the target point for the next step $P_{\mathrm{di}}+1$ along the desired curve according to the specified acceleration or feed rate. 


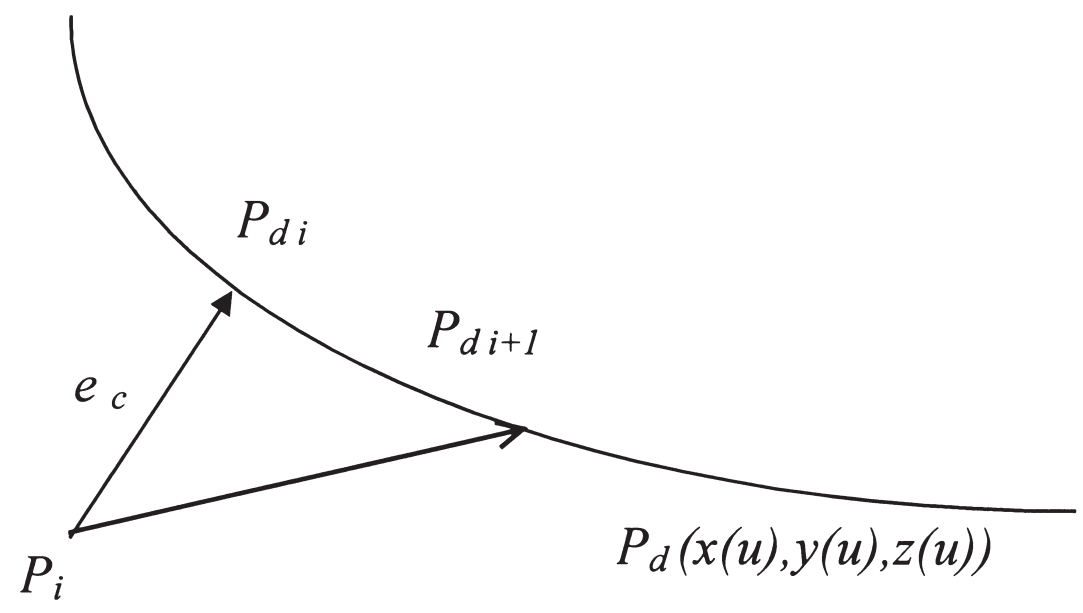

Fig. 1. Geometry of the contour error.

\subsection{Finding the contour error $\mathrm{e}_{c}$}

Given a parametric curve that is represented as, $P_{\mathrm{d}}(x(u), y(u), z(u))$, where $u \in[0, L]$. The tangential vector is defined by $t=\mathrm{d} P_{\mathrm{d}} / \mathrm{d} u$. Assuming that the projection of a point in the $3 \mathrm{D}$ space, say $P_{\mathrm{i}}\left(x_{\mathrm{i}}, y_{\mathrm{i}}, z_{\mathrm{i}}\right)$, to this curve is $P_{\mathrm{di}}\left(x\left(u_{\mathrm{i}}\right), y\left(u_{\mathrm{i}}\right), z\left(u_{\mathrm{i}}\right)\right)$, in which $u_{\mathrm{i}}$ is obtained by solving $\left(P_{\mathrm{di}}-P_{\mathrm{i}}\right) \cdot t=$ 0 . Then, the contour error is the distance between $P_{\mathrm{i}}$ and $P_{\mathrm{di}}$.

$$
\left|e_{\mathrm{c}}\right|=\left|P_{\mathrm{di}}-P_{\mathrm{i}}\right|=\sqrt{\left(x\left(u_{\mathrm{i}}\right)-x_{\mathrm{i}}\right)^{2}+\left(y\left(u_{\mathrm{i}}\right)-y_{\mathrm{i}}\right)^{2}+\left(z\left(u_{\mathrm{i}}\right)-z_{\mathrm{i}}\right)^{2}}
$$

\subsection{The on-line interpolator}

Consider the same curve in Fig. 1, and allow $V(u)$ to be the desired velocity vector along this curve. $V(u)$ is defined by

$$
\begin{aligned}
& V(u)=\frac{\mathrm{d} P_{\mathrm{d}}}{\mathrm{d} t}=\frac{\mathrm{d} P_{\mathrm{d}}}{\mathrm{d} u} \frac{\mathrm{d} u}{\mathrm{~d} t}=\left(\frac{\mathrm{d} x}{\mathrm{~d} u} \overleftarrow{i}+\frac{\mathrm{d} y}{\mathrm{~d} u} \overleftarrow{j}+\frac{\mathrm{d} z}{\mathrm{~d} u} \overleftarrow{k}\right) \frac{\mathrm{d} u}{\mathrm{~d} t}, \\
& |V(u)|=\left|\frac{\mathrm{d} P_{\mathrm{d}}}{\mathrm{d} u}\right| \frac{\mathrm{d} u}{\mathrm{~d} t} .
\end{aligned}
$$

Assume that one specifies the acceleration $|A(u)|$, which is defined by

$$
|A(u)|=\frac{\mathrm{d}|V(u)|}{\mathrm{d} t}=\frac{\mathrm{d}|V(u)|}{\mathrm{d} u} \frac{\mathrm{d} u}{\mathrm{~d} t} .
$$

From Eq. (3) 


$$
|A(u)|=\frac{\mathrm{d}|V(u)|}{\mathrm{d} u} \frac{|V(u)|}{\left|\mathrm{d} P_{d} / \mathrm{d} u\right|}
$$

Hence,

$$
\left.\int_{u_{0}}^{u}\left|A\left(u^{\prime}\right)\right| \frac{\mathrm{d} P_{\mathrm{d}}}{\mathrm{d} u^{\prime}} \mid \mathrm{d} u^{\prime}\right)^{1 / 2}=\int_{V\left(u_{0}\right)}^{V(u)}\left|V\left(u^{\prime}\right)\right| \mathrm{d}\left|V\left(u^{\prime}\right)\right| .
$$

According to Huang and Yang [7],

$$
|V(u)|=\left[\left|V^{2}\left(u_{0}\right)\right|+2 \int_{u_{0}}^{u}\left|A\left(u^{\prime}\right)\right|\left|\frac{\mathrm{d} P_{\mathrm{d}}}{\mathrm{d} u^{\prime}}\right| \mathrm{d} u^{\prime}\right]^{1 / 2} .
$$

From Eq. (3) we have

$$
\int_{u_{0}}^{u} \frac{\left|\mathrm{d} P_{\mathrm{d}} / \mathrm{d} u^{\prime}\right|}{\left|V\left(u^{\prime}\right)\right|} \mathrm{d} u=\int_{t_{0}}^{t} \mathrm{~d} t
$$

Combine Eq. (7) and Eq. (8), and let $u_{0}=u_{\mathrm{i}}, u=u_{\mathrm{i}+1}, t_{0}=t, t=t_{\mathrm{i}+1}, P_{\mathrm{di}+1}$ can be obtained. Then the reference inputs at the time $t_{\mathrm{i}+1}$ is set to $P_{\mathrm{di}+1}-P_{\mathrm{i}}$.

\section{Decoupled dynamics}

After getting the normal feedback signal and the feed rate reference point, one needs to find the system structure to carry out separate normal and tangential controller design. Consider for example a $x$-y table, the dynamics of $x$ and $y$-axes are $g_{11}(s)$ and $g_{22}(s)$, respectively, i.e.

$$
\left[\begin{array}{l}
y_{x}(s) \\
y_{y}(s)
\end{array}\right]=\left[\begin{array}{cc}
g_{11}(s) & 0 \\
0 & g_{22}(s)
\end{array}\right]\left[\begin{array}{l}
u_{x}(s) \\
u_{y}(s)
\end{array}\right]=\mathbf{G}(s)\left[\begin{array}{l}
u_{x}(s) \\
u_{y}(s)
\end{array}\right]
$$

To obtain the dynamics in the tangential and the normal directions to the desired trajectory, one proceeds by defining a transformation matrix, $\mathbf{F}$, between the errors in the $x, y$ directions, $e_{x}$ and $e_{y}$, and the errors in the tangential and normal directions, $e_{t}$ and $e_{n}$. (As shown in Fig. 2.) Thus $\mathbf{F}$ can be expressed as

$$
\mathbf{F}=\left[\begin{array}{ll}
f_{11} & f_{12} \\
f_{21} & f_{22}
\end{array}\right]=\left[\begin{array}{cc}
\cos \theta & \sin \theta \\
-\sin \theta & \cos \theta
\end{array}\right],
$$

where $\theta$ is the angle between the $x$-axis and the tangent of the desired trajectory. Therefore,

$$
\left[\begin{array}{l}
y_{t}(s) \\
y_{n}(s)
\end{array}\right]=\mathbf{F}\left[\begin{array}{l}
y_{x}(s) \\
y_{y}(s)
\end{array}\right]=\mathbf{F}^{*} \mathbf{G}\left[\begin{array}{l}
u_{x}(s) \\
u_{y}(s)
\end{array}\right] .
$$




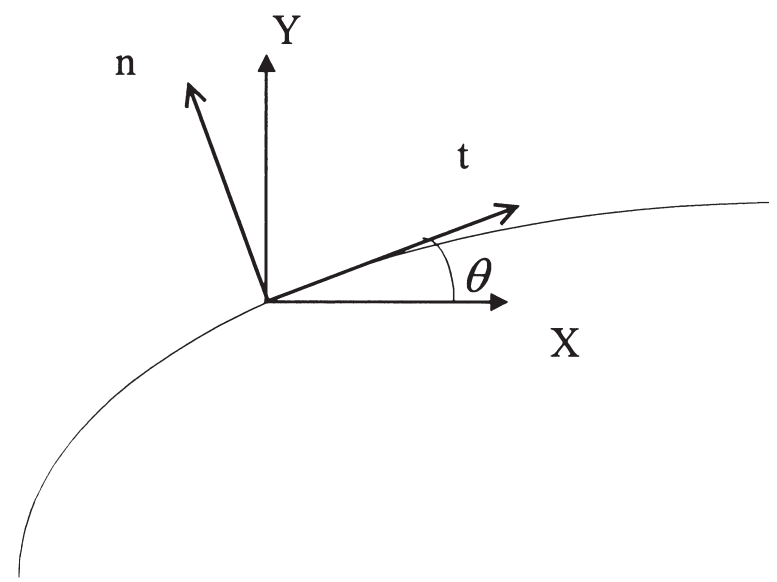

Fig. 2. Geometry of $x-y$ coordinate and tangential-normal coordinate.

In order to decouple the system dynamics into tangential and normal directions, define a decoupling matrix which acts like a filter between $\left[\begin{array}{l}u_{t}(s) \\ u_{n}(s)\end{array}\right]$ and $\left[\begin{array}{l}u_{x}(s) \\ u_{y}(s)\end{array}\right]$, i.e.

$$
\left[\begin{array}{l}
u_{x}(s) \\
u_{y}(s)
\end{array}\right]=\left[\begin{array}{ll}
c_{11}(s) & c_{12}(s) \\
c_{21}(s) & c_{22}(s)
\end{array}\right]\left[\begin{array}{l}
u_{t}(s) \\
u_{n}(s)
\end{array}\right]=\mathbf{C}\left[\begin{array}{l}
u_{t}(s) \\
u_{n}(s)
\end{array}\right]
$$

Substitute Eq. (10) into Eq. (9)

$$
\left[\begin{array}{l}
y_{t}(s) \\
y_{n}(s)
\end{array}\right]=\mathbf{F G C}\left[\begin{array}{l}
u_{t}(s) \\
u_{n}(s)
\end{array}\right]=\mathbf{G}_{t}\left[\begin{array}{l}
u_{t}(s) \\
u_{n}(s)
\end{array}\right]
$$

If there exists a $\mathbf{C}$ that diagonalizes $\mathbf{G}_{t}$, then the system dynamics can be decoupled into

$$
\mathbf{G}_{t}(s)=\left[\begin{array}{cc}
g_{t_{11}}(s) & 0 \\
0 & g_{t_{22}}(s)
\end{array}\right] .
$$

From Eq. (11),

$$
\begin{aligned}
& {\left[\begin{array}{cc}
g_{t_{11}} & 0 \\
0 & g_{t_{22}}
\end{array}\right]=\left[\begin{array}{ll}
f_{11} & f_{12} \\
f_{21} & f_{22}
\end{array}\right]\left[\begin{array}{cc}
g_{11} & 0 \\
0 & g_{22}
\end{array}\right]\left[\begin{array}{ll}
c_{11} & c_{12} \\
c_{21} & c_{22}
\end{array}\right],} \\
& =\left[\begin{array}{ll}
f_{11} g_{11} & f_{12} g_{22} \\
f_{21} g_{11} & f_{22} g_{22}
\end{array}\right]\left[\begin{array}{ll}
c_{11} & c_{12} \\
c_{21} & c_{22}
\end{array}\right]
\end{aligned}
$$

or, 


$$
\left\{\begin{array}{c}
g_{t_{11}}=f_{11} g_{11} c_{11}+f_{12} g_{22} c_{21} \\
0=f_{11} g_{11} c_{12}+f_{12} g_{22} c_{22} \\
0=f_{21} g_{11} c_{11}+f_{22} g_{22} c_{21} \\
g_{t_{22}}=f_{21} g_{11} c_{12}+f_{22} g_{22} c_{22}
\end{array}\right.
$$

Solving Eq. (14), we get

$$
\mathbf{C}=\left[\begin{array}{cc}
\frac{f_{22}}{\left(f_{11} f_{22}-f_{12} f_{21}\right)} \frac{g_{t_{11}}}{g_{11}} & -\frac{f_{12}}{\left(f_{11} f_{22}-f_{12} f_{21}\right)} \frac{g_{t_{22}}}{g_{11}} \\
-\frac{f_{21}}{\left(f_{11} f_{22}-f_{12} f_{21}\right)} \frac{g_{t_{11}}}{g_{22}} & \frac{f_{11}}{\left(f_{11} f_{22}-f_{12} f_{21}\right)} \frac{g_{t_{22}}}{g_{22}}
\end{array}\right]
$$

C must satisfy some constraints:

1. C must be stable to avoid internally unstable. Therefore,

- $g_{t_{11}}$ must have stable poles.

- $g_{t_{11}}$ must contain all the non-minimal phase zeros of $g_{11}$ 's and $g_{22}$ 's.

- $g_{t_{22}}$ must have stable poles.

- $g_{t_{22}}$ must contain all the non-minimal phase zeros of $g_{11}$ 's and $g_{22}$ 's.

2. $\mathbf{C}$ must be realizable. Therefore,

- The relative degrees of $g_{t_{11}}$ must be greater than the higher relative degrees of $g_{11}$ and $g_{22}$.

- The relative degrees of $g_{t_{22}}$ must be greater than the higher relative degrees $g_{11}$ and $g_{22}$.

From Eq. (15), one obtains a dynamically decoupled system in the form of Eq. (13), whose dynamics are decoupled into a tangential and a normal (contouring) directions according to the desired contour. The block diagram for system design thus becomes as shown in Fig. 3. With this setup, conceptually, the decoupled controller, $\mathbf{K}(s)$, design can be carried out in the tangential and normal directions. The actual controller, $\mathbf{K}(s)$, to be implemented, however, is coupled and is nonlinear as shown in Fig. 4.

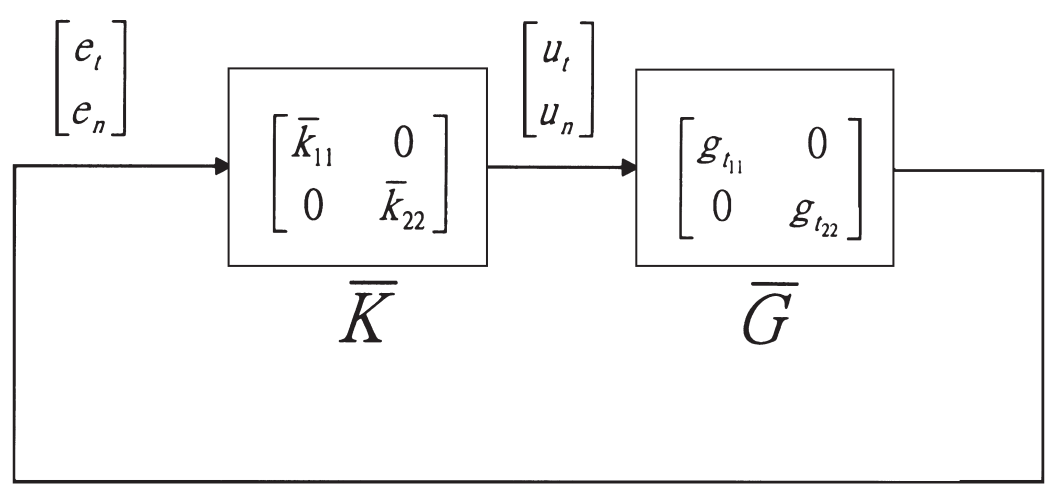

Fig. 3. Equivalent block diagram of decoupled control structure. 


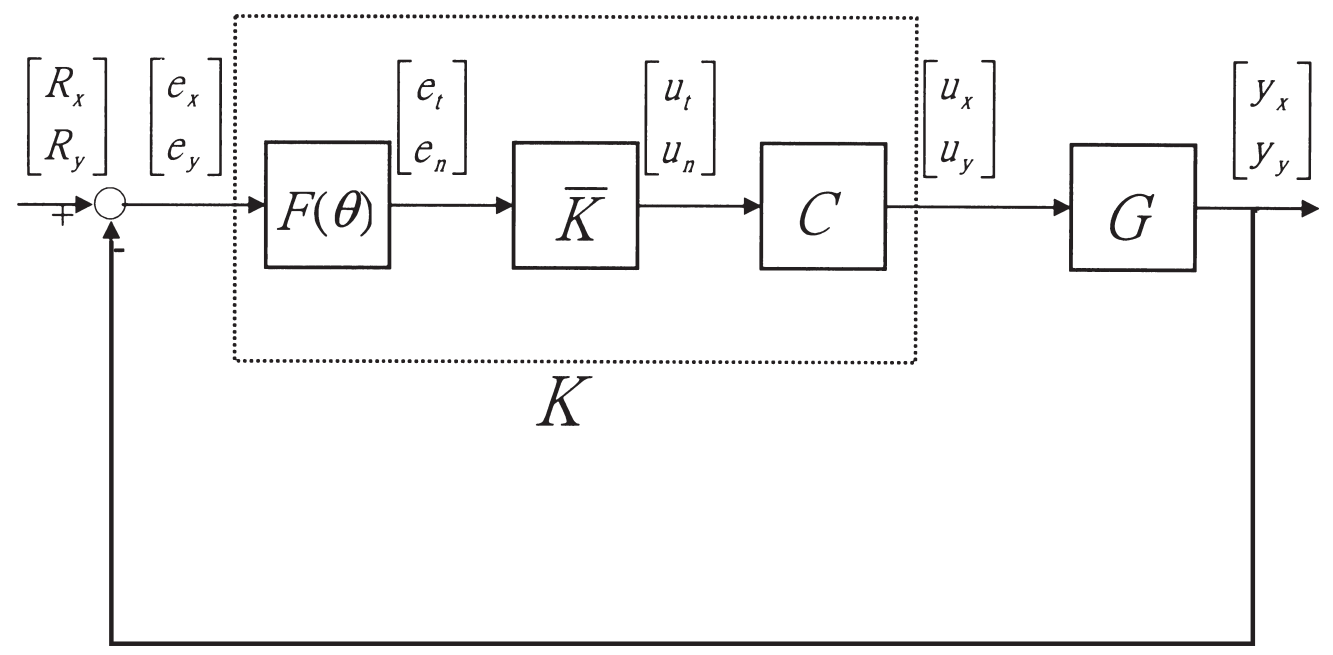

Fig. 4. Real block diagram of decoupled control structure.

\section{Experiment setup and test example}

Fig. 5 presents the experiment $x-y$ table. The table is $850 \mathrm{~kg}$ in weight and the strokes of $x$ and $y$-axes are 400 and $300 \mathrm{~mm}$. The measurement resolution in both axes is about $10 \mu \mathrm{m}$. Two

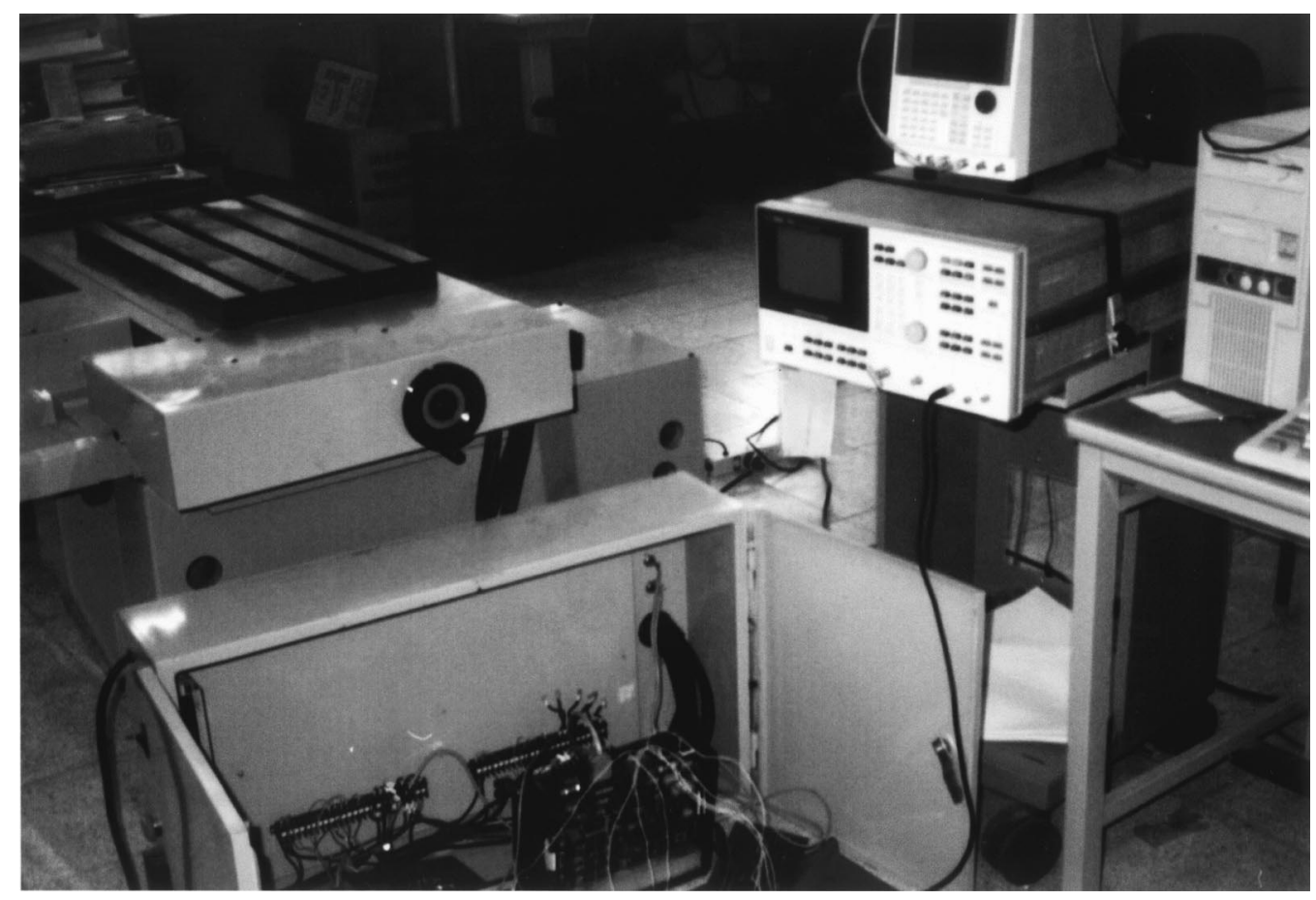

Fig. 5. Control system diagram. 
AC servomotors each drive a ball-screw to position the $x-y$ table. The resolvers mounted on the end of the servomotors measure the position of the table. A counter board in the control computer takes the position counts. The control computer, a 486-based PC, calculates the errors and computes the control effort.

As an illustration example, consider a circular path as shown in Fig. 6. Each point on the path can be represented by

$$
P_{\mathrm{d}}(x(u), y(u))=P_{\mathrm{d}}(r \cos u, r \sin u) .
$$

The tangent direction at point $P_{d}$ is

$$
t=\frac{d P_{d}}{d u}=(-r \sin u, r \cos u)
$$

Following from Section 2, we have

$$
\begin{aligned}
& \left(P_{\mathrm{d}}-P_{\mathrm{i}}\right) \cdot t=0 \\
& \left(r \cos u-x_{\mathrm{i}}, r \sin u-y_{\mathrm{i}}\right) \cdot(-r \sin u, r \cos u)=0 \\
& x_{\mathrm{i}} \sin u=y_{\mathrm{i}} \cos u \\
& u_{\mathrm{i}}=\tan ^{-1}\left(\frac{y_{\mathrm{i}}}{x_{\mathrm{i}}}\right)
\end{aligned}
$$

and

$$
P_{\mathrm{di}}=\left(r \cos \left(\arctan \left(\frac{y_{\mathrm{i}}}{x_{\mathrm{i}}}\right)\right), r \sin \left(\arctan \left(\frac{y_{\mathrm{i}}}{x_{\mathrm{i}}}\right)\right)\right) .
$$

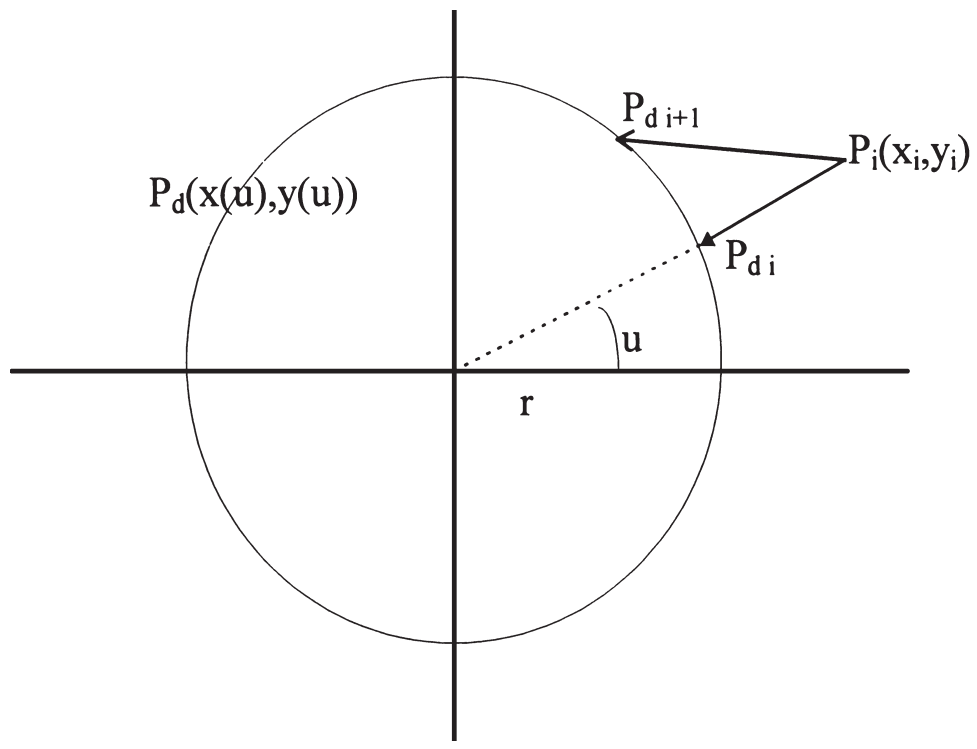

Fig. 6. Circular path command generation. 
The contour error in the normal direction is thus,

$$
\left|e_{\mathrm{c}}\right|=\left|P_{\mathrm{i}}-P_{\mathrm{ch}}\right|=\sqrt{\left(x_{\mathrm{i}}-r \cos \left(\tan ^{-1}\left(\frac{y_{\mathrm{i}}}{x_{\mathrm{i}}}\right)\right)\right)^{2}+\left(y_{\mathrm{i}}-r \sin \left(\tan ^{-1}\left(\frac{y_{\mathrm{i}}}{x_{\mathrm{i}}}\right)\right)\right)^{2}} .
$$

If we specify the acceleration $|A(u)|$ and the maximum allowable feed rate $F$ for a trapezoidal velocity profile, then

$$
|A(u)|=\left\{\begin{array}{l}
A \forall V\left(u_{\mathrm{i}+1}\right)<F(\text { Feedrate }) \\
0 \forall V\left(u_{\mathrm{i}+1}\right) \geq F(\text { Feedrate })
\end{array}\right.
$$

$u_{\mathrm{i}+1}=\frac{A T^{2}}{2}+\frac{T V\left(u_{\mathrm{i}}\right)}{r}+u_{\mathrm{i}}$, where $T$ is sampling period and

$$
V\left(u_{\mathrm{i}+1}\right)=\left[V^{2}\left(u_{\mathrm{i}}\right)+2 \operatorname{Ar}\left(u_{\mathrm{i}+1}-u_{\mathrm{i}}\right)\right]^{1 / 2}
$$

under condition that

$$
V\left(u_{\mathrm{i}+1}\right) \geq F,\left\{\begin{array}{c}
u_{\mathrm{i}+1}=\frac{T F}{r}+u_{\mathrm{i}} \\
V\left(u_{\mathrm{i}+1}\right)=F
\end{array}\right.
$$

Therefore,

$$
P_{\mathrm{di}+1}=\left(r \cos \left(u_{t+1}\right), r \sin \left(u_{\mathrm{i}+1}\right)\right) .
$$

Finally, the reference input is

$$
R_{\mathrm{i}+1}=P_{\mathrm{di}+1}-P_{\mathrm{i}}=\left(r \cos \left(u_{\mathrm{i}+1}\right)-x_{\mathrm{i}}, r \sin \left(u_{\mathrm{i}+1}\right)-y_{\mathrm{i}}\right)
$$

After getting the reference input, the normal and tangential controllers with traditional design algorithms can be designed separately.

In this example, we use the MATLAB Nonlinear Control Toolbox to find a non-overshoot controller for the nominal control. No overshoot in the normal direction means non-overcutting in the machining process. Because the decoupling is obtained for every position, this controller will achieve no overcutting throughout the trajectory.

\section{Results and discussion}

Fig. 7(a-d) show the experimental results of tracking a $20 \mathrm{~mm}$ circle with cross-coupled control. The feed rate in this case is set to $20 \mathrm{~mm} / \mathrm{s}$. Fig. 8(a-d) were obtained under the same test condition as Fig. 7 except that the cross-coupled controller gain was doubled. It can be seen that the contour error is reduced. It is to be noticed that the tracking speed increases slightly. This is due to the fact that the cross-coupled control input becomes larger so that the drives move faster not only in the contouring direction but also in the tangential direction. By further increasing the 
(a)

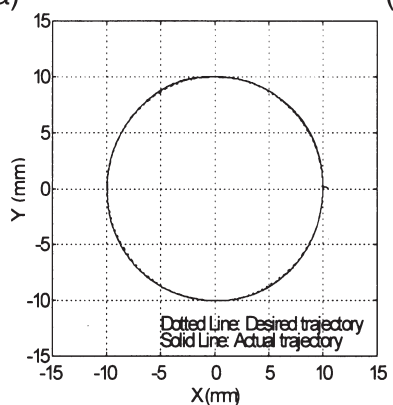

(b)

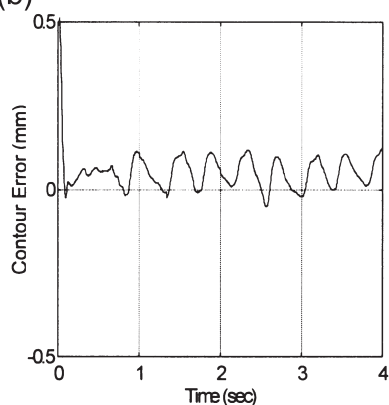

(c)

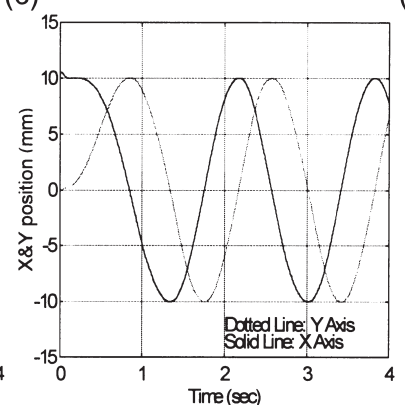

(d)

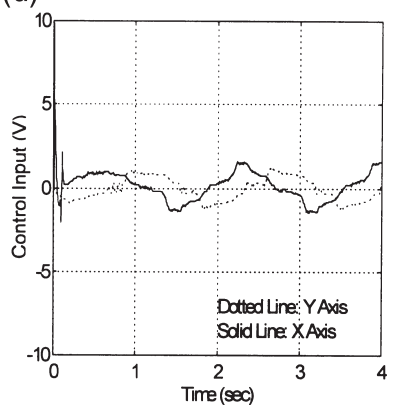

Fig. 7. Cross-coupled controller acting with reference feed rate $=20 \mathrm{~mm} / \mathrm{s}$. (a) $x-y$ plot; (b) $x$ and $y$ position with time; (c) contour error; (d) $x$ and $y$ control inputs.

(a)

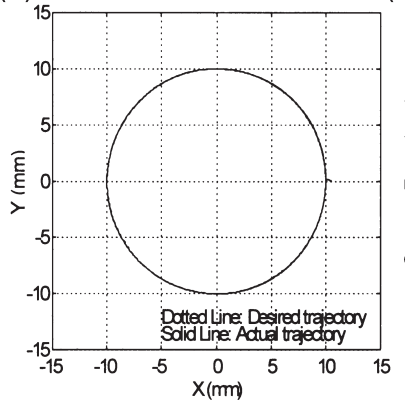

(b)

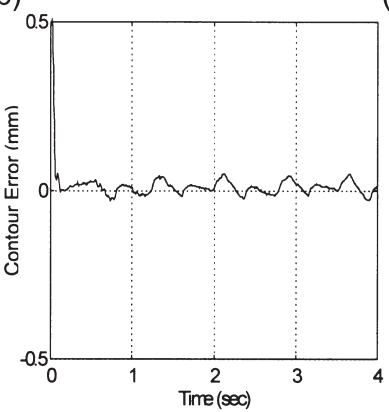

(c)

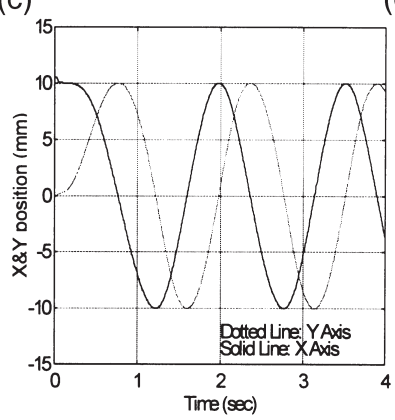

(d)

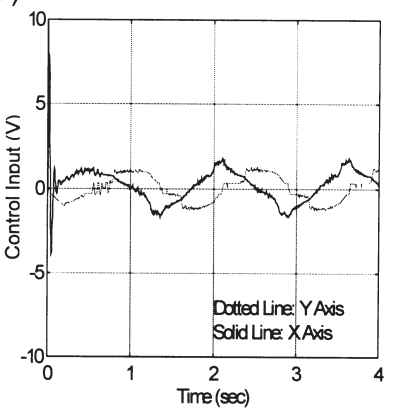

Fig. 8. Cross-coupled controller acting with reference feed rate $=20 \mathrm{~mm} / \mathrm{s}$ and cross-coupled control input gain doubled. (a) $x-y$ plot; (b) $x$ and $y$ position with time; (c) contour error; (d) $x$ and $y$ control inputs.

cross-coupled controller gain to three times (Fig. 9), it is seen that, although the contour error decreases a little further, it starts to cause undesirable chattering.

Fig. 10 shows the results of the decoupled controller proposed in this paper. The command generation procedure is the same as that in Figs. 7-9. It is seen that the contour error is in the

(a)

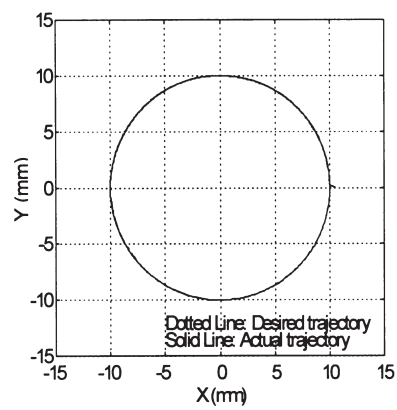

(b)

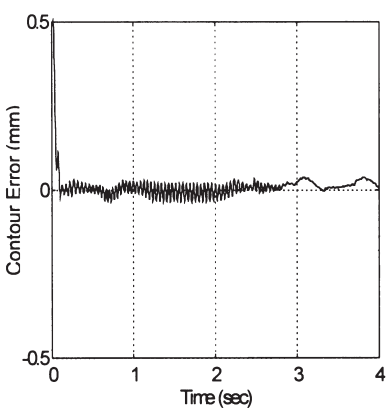

(c)

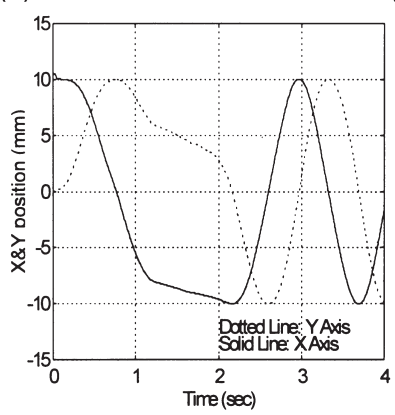

(d)

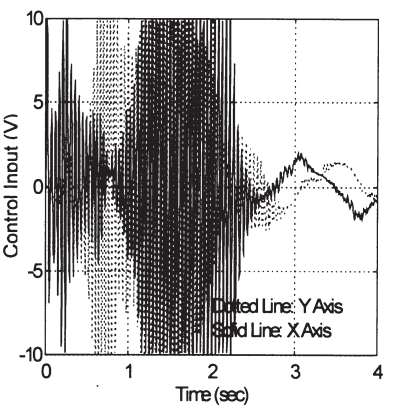

Fig. 9. Cross-coupled controller acting with reference feed rate $=20 \mathrm{~mm} / \mathrm{s}$ and cross-coupled control input gain tripled. (a) $x-y$ plot; (b) $x$ and $y$ position with time; (c) contour error; (d) $x$ and $y$ control inputs. 
(a)

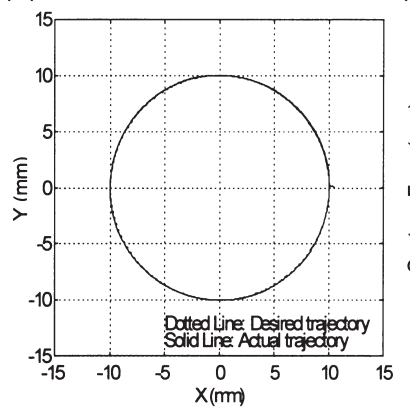

(b)

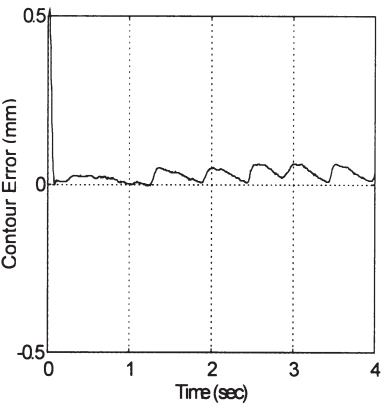

(c)

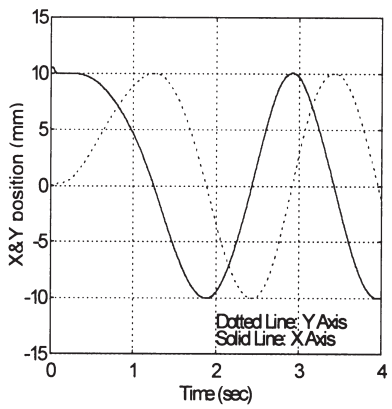

(d)

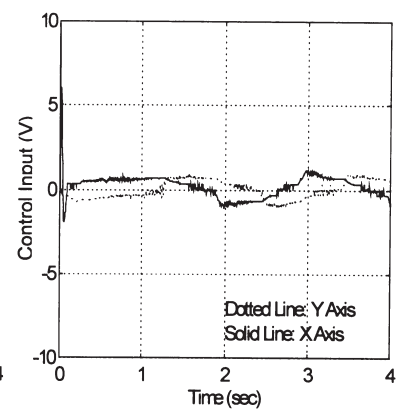

Fig. 10. Decoupled controller acting with reference feed rate $=20 \mathrm{~mm} / \mathrm{s}$. (a) $x-y$ plot; (b) $x$ and $y$ position with time; (c) contour error; (d) $x$ and $y$ control inputs.

range of that of Fig. 8 and better than that of Fig. 7. Moreover, it is very successful in avoiding overcutting, which was part of the original goal in the normal controller. Actually, because of the non-overshoot criterion, the response is slower than that in Figs. 7 and 8. We can obtain similar results when the reference feed rate increases to $40 \mathrm{~mm} / \mathrm{s}$.

\section{Conclusions}

This paper proposed a new path following controller with decoupled tangential and the normal control. The controller de-couples the tracking system into normal contour error dynamics and tangential speed regulation dynamics. The normal control is responsible for maintaining the tool on the target trajectory, and the tangential control will move the tool along the trajectory at the desired speed. Since the errors must be calculated on-line, an on-line interpolator is introduced in the controller. This approach makes the new controller more robust to system delays because the on-line command generator is not affected by the delay caused by different material hardness or path obstacles. With the advanced computer speed, doing so during each sampling period does not seem to have any drawbacks.

The new decoupled controller can be regarded as a modification to the cross-coupled controller. This approach maintains the advantages of the cross-coupled controller, while clearly identifying the control effort to drive the tool along the desired trajectory. Thus, the new controller allows for a distinct trajectory regulation and feed rate specifications. The experimental results demonstrated that the decoupled controller is capable of achieving a separate trajectory following feed rate requirements. In addition, the new controller also achieves non-overshoot control to avoid overcutting.

\section{Acknowledgement}

This work was sponsored by the National Science Council, ROC under project No. 85-2212E-002-060. 


\section{References}

[1] Y. Koren, Design of computer control for manufacturing systems, ASME Journal of Engineering for Industry 101 (1979) 326-332.

[2] P. Sarachik, J.R. Ragazzini, A two dimensional feedback control system, Transactions AIEE 76 (II) (1957) 55-61.

[3] Y. Koren, Cross-coupled biaxial computer control for manufacturing systems, ASME Journal of Dynamic Systems, Measurement, and Control 102 (1980) 265-272.

[4] Y. Koren, C.C. Lo, Advanced controllers for feed drives, Annals of the CIRP 41-2 (1992) 686-698.

[5] T.C. Tsao, M. Tomizuka, Adaptive zero phase error tracking algorithm for digital control, ASME Journal of Dynamic Systems, Measurement and Control 109 (1988) 349-354.

[6] J. Bulter, B. Haack, M. Tomizuka, Reference generation for high speed coordinated motion of a two axis system, ASME Journal of Dynamic Systems, Measurement and Control 13 (1) (1991) 67-74.

[7] M. Tomizuka, J.S. Hu, T.C. Chiu, T. Kamano, Synchronization of two motion control axes under adaptive feedforward control, ASME Journal of Dynamic Systems, Measurement and Control 114 (1992) 196-203.

[8] P.K. Kulkarni, K. Srinivasan, Optimal contouring control of multi-axial feed drive servomechanisms, ASME Journal of Engineering for Industry 111 (1989) 140-148.

[9] P.K. Kulkarni, K. Srinivasan, Cross-coupled control of biaxial feed drive servomechanisms, ASME Journal of Dynamic Systems, Measurement and Control 112 (2) (1990) 225-232.

[10] Y. Koren, C.-C. Lo, Variable-gain cross-coupling controller for contour, Annals of the CIRP 40-1 (1991) 371-373.

[11] H.Y. Chuang, C.H. Liu, Cross-coupled adaptive feedrate control for multiaxis machine tools, ASME Journal of Dynamic Systems, Measurement and Control 106 (1991) 451-457.

[12] O. Masory, Y. Koren, Reference word circular interpolators for CNC systems, ASME Journal of Engineering for Industry 104 (1982) 400-405.

[13] J.T. Huang, D.C.H. Yang, Precision command generation for computer controlled machines. Precision Machining: Technology and Machine Development and Improvement, PED_Vol. 58 ASME (1992).

[14] R. Stadelmann, Computation of nominal path values to generate various special curves for machine, Annals of the CIRP 38 (1) (1989) 373-376. 